\title{
Application of Helium Ion Microscope for Sample Modification at Nanoscale
}

\author{
Maria Rudneva ${ }^{1}$, Emile van Veldhoven ${ }^{2}$, Sairam K. Malladi ${ }^{1}$ and Henny W. Zandbergen ${ }^{1}$ \\ 1. Kavli Institute of Nanoscience, National Centre for High Resolution Electron Microscopy, \\ Delft University of Technology, Delft, the Netherlands \\ ${ }^{2}$. Van Leeuwenhoek Laboratory, TNO, Delft, the Netherlands
}

Amid the variety of TEM sample preparation techniques, the use of a charged particles beam seems to be one of the most promising from the perspective of modification and sculpting of structures at the nanoscale. Application of electron, argon or gallium ion beams for such modifications has already been studied quite well in the past decades. Recently the use of a helium beam for this purpose has become possible in a helium ion microscope (HeIM) developed by Carl Zeiss AG.

The outstanding performance of the present state of the art high-resolution electron microscopes demands for artifact-free sample preparation methods and HeIM modification could possibly satisfy this requirement and provide defect-free electron transparent specimens.

This contribution presents the applicability of the HeIM for precise modification of thin $\mathrm{Si}, \mathrm{Cu}$, $\mathrm{Pt}, \mathrm{Au}$ and $\mathrm{SrTiO}_{3}$ samples and subsequent analysis of them in transmission electron microscope (TEM). Possibility to combine such focused helium ion beam modification with local heating is also discussed.

Firstly, 15nm thick Pt bridges of standard geometry deposited on $100 \mathrm{~nm}$ thick SiN membrane were sculptured into various shapes by HeIM (Figure 1). Simultaneously the membrane was selectively removed around the bridges. Inspection on the modified samples with TEM shows preservation of crystallinity of the sample up to the edge of the cut indicating that artifact free cutting of Pt is possible by HeIM. However comparison of HeIM-modified and initial samples during in situ current induced annealing clearly indicates that He ions have some impact on the speed of the grain growth in the sample.

A special heating stage was made to allow nano-scale sculpting of the samples at various temperatures up to $700^{\circ} \mathrm{C}$. Local heating of the lamella type of specimens was facilitated by using MEMS based heaters developed in-house. The MEMS heaters can be turned on or off by a switch without taking them out of the microscope.

Use of this heating stage made it possible to expand the area of HeIM application for TEM samples preparation. For instance, it was previously reported that room temperature cutting of Si with focused He-ion beam leads to formation of lattice defects, big bubbles on the specimen and even to complete amorphisation. We prepared a few Si lamellas of different thicknesses: 100 $300 \mathrm{~nm}$ by Ga ion FIB and transferred them to MEMS heaters. Next, samples were sculpted locally with a focused He-ion beam at both room and elevated temperatures. The detailed analysis of the modified samples was carried out with FEI Titan TEM operated at $300 \mathrm{kV}$. Comparison of diffraction patterns obtained from the illuminated area for a $100 \mathrm{~nm}$ and $300 \mathrm{~nm}$ thick samples makes evident that some lattice deformation was created in $300 \mathrm{~nm}$ thick sample 
during room temperature milling. Absence of visible defects in samples with thicknesses up to $200 \mathrm{~nm}$ can be explained by good collimation of the helium beam at these thicknesses. Milling at higher temperatures $\left(\sim 600^{\circ} \mathrm{C}\right)$ resulted in cuts with crystalline edges for both thin and thick samples (Figure 2). This difference can be explained by increased out-diffusion of helium from the sample at elevated temperature.

A staircase type of cutting as well as tilting of the stage during gives a possibility to use HeIM for creating a wedge shaped sample for the investigation of the change of in the image as a function of thickness. We carried out a detailed study on the influence of focused helium beam on $\mathrm{SrTiO}_{3}$ sample. It was shown that room temperature modification leads to defect formation whereas heating of the sample up to $550^{\circ} \mathrm{C}$ provides thin artifact-free area without a significant amorphous surface layer

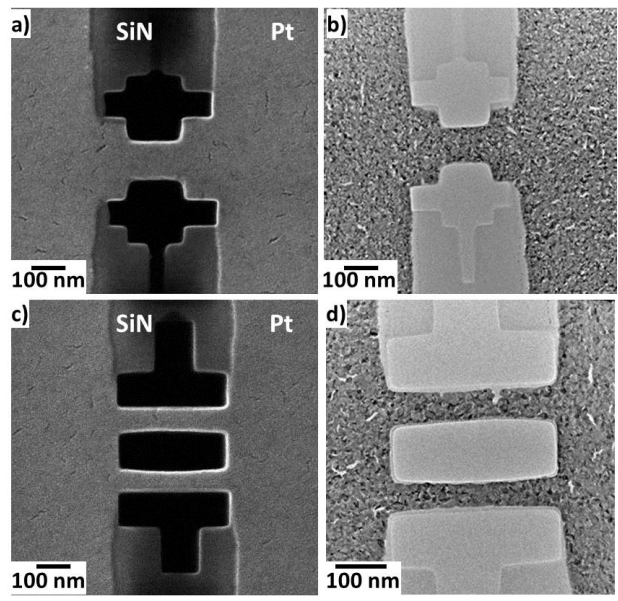

Figure 1. $15 \mathrm{~nm}$ thick platinum bridges on $\mathrm{SiN}$ membrane modified with focused He-ion beam. (a), (c) - images obtained in HeIM; (b), (d) - images obtained in TEM.
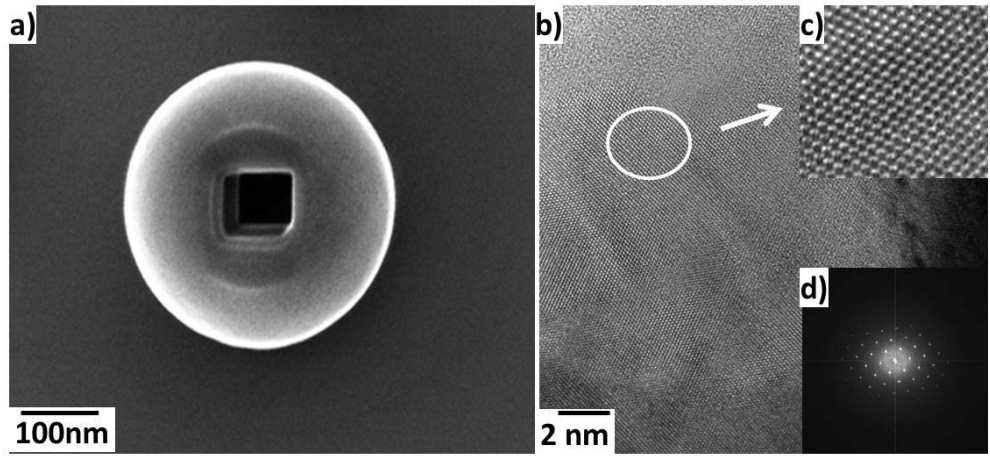

Figure 1. Result of modification of $300 \mathrm{~nm}$ thick Si sample with focused He-ion beam: (a) room temperature cutting leads to formation of big white halo (bubble) around the area of cut; (b) cutting at $600^{\circ} \mathrm{C}$ allows to obtain thin area without any noticeable defect, that confirmed by high resolution TEM image (c) and diffraction patterns (d) from illuminated area.

References:

[1] M Rudneva et al, MRS Proceedings 1455 (2012) 